\title{
Propuesta de mujeres campesinas para los agronegocios lácteos en Bolívar-Ecuador
}

\section{Proposal of rural women for dairy agribusiness in Bolivar- Ecuador}

\author{
Iván Alberto Analuisa Aroca ${ }^{1,2}$ https://orcid.org/0000-0002-3798-3122 \\ ${ }^{1}$ Universidad Técnica de Manabi, Portoviejo, Ecuador \\ ${ }^{2}$ Universidad de Córdoba, Córdoba, España \\ ivan.analuisa@utm.edu.ec
}

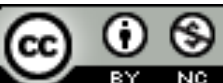

Esta obra está bajo una licencia internacional Creative Commons Atribución-NoComercial 4.0.

$\begin{array}{ll}\text { Enviado: } & 2021 / 10 / 31 \\ \text { Aceptado: } & 2021 / 12 / 07 \\ \text { Publicado: } & 2021 / 12 / 30\end{array}$

\section{Resumen}

La propuesta de crear una quesera en el sector rural del cantón Guaranda, en la provincia de Bolívar-Ecuador, tuvo como objetivo diseñar un modelo de agronegocios para las mujeres campesinas de dicho sector. Esta iniciativa se realizó con la intención de fortalecer la cadena de valor de la leche y generar así confianza en las mujeres campesinas del cantón, y, al mismo tiempo, reactivar su economía. En el desarrollo de esta propuesta, se realizaron encuestas para levantar información orientada a conocer las necesidades de la población, $\mathrm{y}$, con ello, generar información base que permita identificar la oferta y demanda de productos agrícolas. Además, se organizaron encuentros con dirigentes y mujeres campesinas con miras a la implementación de una organización empresarial. En base a los resultados obtenidos, se determinó la existencia de una demanda insatisfecha de queso en el mercado y se conformaron aglomerados para determinar las variables significativas del estudio de mercado. Los resultados de la investigación también permitieron plantear una propuesta de producción de 200 kilos de queso/día de diferentes tipos, como mozzarella y andino. El modelo de agronegocios propuesto, según la evaluación financiera que se realizó, tiene una alta rentabilidad para los inversionistas y un alto impacto social en la economía rural. Cabe enfatizar que la mujer campesina ha sido considerada como el componente principal del entorno empresarial de este proyecto, pues constituye un actor clave de las actividades primarias y productivas de la economía familiar del sector rural de este cantón.

Palabras clave: Bolívar, emprendimientos, mujeres, queso, reactivación.

Sumario: Introducción, Materiales y Métodos, Metodología, Resultados y Discusión y Conclusiones.

Como citar: Analuisa, I. (2021). Propuesta de mujeres campesinas para los agronegocios lácteos en Bolívar-Ecuador. Revista Tecnológica - Espol, 33(3), 152-166.

http://www.rte.espol.edu.ec/index.php/tecnologica/article/view/873 


\begin{abstract}
The proposal to create a cheese factory in the rural sector of the Guaranda canton, in the province of Bolivar- Ecuador, was aimed at designing an agribusiness model for rural women in that sector. This initiative was carried out with the intention of strengthening the milk value chain and thus generating confidence in the rural women of the canton and, at the same time, reactivating their economy. In the development of this proposal, surveys were conducted to gather information on the needs of the population, and thus generate baseline information to identify the supply and demand of agricultural products. In addition, meetings were organize with leaders and women farmers with a view to implementing a business organization. Based on the results, it was determined the existence of unsatisfied demand for cheese in the market and clusters were formed to determine the significant variables of the market study. The results of the research also made it possible to propose a production f 200 kilos of cheese / day of different types of cheese, such as mozzarella and Andean. The proposed agribusiness model, according to the financial evaluation, is highly profitable for investors and has a high social impact on the rural economy. It should be emphasized that rural women have been considered as the main component of the business environment of this project, as they are key players in the primary and productive activities of the family economy in the rural sector of this canton.
\end{abstract}

Keywords: Bolívar, entrepreneurship, women, cheese, reactivation.

\title{
Introducción
}

Las tareas de identificación, planificación, evaluación, seguimiento y control de ideas de proyectos, junto a programas de desarrollo, se enmarcan en los planes de desarrollo estratégico, los mismos que permiten orientar acciones integrales para mejorar las condiciones de vida de la población en sus diferentes niveles de organización: local, municipal, zonal y nacional. Los gobiernos locales deben exponer de manera clara y explícita los objetivos estratégicos dentro de su planificación, acorde a las prioridades básicas de la localidad. Esto con el fin de obtener los recursos económicos o asignaciones presupuestarias otorgadas por el Gobierno central. Los procesos de planificación sirven como referencia clave para evaluar la gestión de los Gobiernos locales, pues estos permiten comparar los resultados que se han obtenido durante la gestión y los objetivos planteados en su planificación anual, sobre todo, en relación a la solución de los problemas detectados en un determinada comunidad (Generalitat Valenciana, 2017).

Por otro lado, en el campo de los negocios se desarrollan estrategias de producción, comercialización y publicidad con la finalidad de posicionar un determinado producto en el mercado (BID, 2004; Ortegón et al., 2015). En el contexto de la gestión de proyectos, los objetivos estratégicos deben ser verificables, por lo que se deben seleccionar indicadores o fuentes apropiadas para tal propósito. Entre los efectos medibles, está el de la capacidad productiva y el del incremento de la productividad entre los agricultores como efecto de la disponibilidad de crédito y de la transferencia de tecnología (Escribano \& Hummel, 2020; Ramírez, 2007).

La proyección de escenarios en el contexto latinoamericano no es fácil, por tanto, es necesario considerar "la construcción de la proximidad en el medio rural" como elemento fundamental para enfrentar, desde lo local, una infinidad de desafíos económicos, productivos y organizativos (Martínez, 2015; OCDE/FAO, 2019).

Actualmente, se puede observar cómo se ha gestado un cambio de roles en los procesos productivos de las cadenas de valor dentro de las comunidades; esto debido a la inclusión de actividades $\mathrm{y}$ acciones encaminados a proponer estrategias inclusivas de negocios, 
mejoramiento e innovación de productos, diseños de canales de distribución, modelos de negocios rentables (sobre todo, en el campo de las mujeres emprendedoras), sentido asociativo en la producción y buenas prácticas agrícolas (Henríquez et al., 2019; Moreno et al., 2020). Según Figueroa y García (2019), se consideran acciones a aquellas actividad en las cual se emprenden nuevos proyectos para transformar el mundo, lo cual requiere de la participación de la ciudadana (Pizaña, Fletes and González, 2019).

En la actualidad, el referente más importante del desarrollo rural se lo encuentra en el liderazgo de jóvenes y mujeres, quienes, desde sus espacios cotidianos, han estado gestando alternativas que les ha permitido mejorar la calidad de vida de sus comunidades (Varisco, 2016). Así también, se debe señalar que el trabajo de mujeres (Barrientos, 2019) e hijos, quienes son los que absorben la mayor carga de cuidados en el sector rural, reducen los costos de la economía familiar y doméstica, pues la fuerza de trabajo de estos no son remunerados (CEPAL., 2020; Dos Santos, 2020). Aunque cabe mencionar también, que las mujeres han tenido un notable aumento en la fuerza laboral remunerada, al emprender en diferentes negocios (Kotler and Armstrong, 2017; Vanoni et al., 2017).

En torno al tema de la posesión de tierras, se debe resaltar que, a partir del programa de redistribución y titulación de propiedad "Plan Tierras" del 2016, las mujeres campesinas del sector han sido beneficiarias de la entrega de 33\% de un total de 60.100 hectáreas y de 11.081 beneficiarios, lo que indica que el MAGAP ha incluido a las mujeres, tanto jefas de hogar, solteras y casadas, en sus planes (Deere, 2018), permitiéndoles obtener derechos a posesión de tierra (Vergara and Kay, 2018). De igual forma, la ayuda financiera reembolsable para promover emprendimientos de mujeres en el denominado "Fondo semilla", apoya actividades productivas en ámbitos como el comercio por catálogo, la producción, la pesca, las artesanías, la venta de abarrotes y la venta de ropa (MAGAP, 2017; Valencia Cruzaty, 2017; INECESPAC, 2020).

Por otra parte, la producción de quesos está considerada como parte de los Objetivos de Desarrollo Sustentable (Territorio Ecuador ODS, 2018), y su implementación forma parte de los diferentes planes nacionales (Arteaga-Cruz, 2017), como el Plan Estratégico de Desarrollo del Turismo Sostenible para el Ecuador. Esto como una estrategia para vincular este tipo de actividades productivas con la cadena de valor del turismo y de los emprendimientos empresariales relacionados con los mecanismos de comercio justo, que fomentan formas de producción sostenibles, entre ellas, la agroecología y agroforestería, agroturismo y ecoturismo. Todas estas modalidades se caracterizan por la conservación de la agrobiodiversidad y promueven la disminución del uso de agroquímicos. En este contexto, las principales actividades que se impulsan son la producción, la comercialización y el consumo de los siguientes productos: a) alimentos básicos orgánicos, como cereales, fruta, hortalizas, semillas y hierbas condimentadas y plantas medicinales, entre otros; b) alimentos orgánicos procesados, como por ejemplo mermeladas, quesos, yogures, vinos de fruta y tés, entre otros (Ministerio de Agricultura y Ganadería, 2019b; Ministerio de Turismo del Ecuador, 2020).

Basados en la forma de construcción de los modelos de gestión administrativa, se pone a consideración la misión y visión empresariales considerando las estrategias empresariales de Kotler y Armstrong (2017) y HubSpot (2019):

La Misión de la empresa es ofrecer productos que brinden altos niveles de calidad, así como cubrir las necesidades calóricas de los consumidores, siendo amigable con la naturaleza. 
La Visión es el posicionamiento de la marca en los segmentos de consumo a nivel nacional, a partir de la oferta de los mejores beneficios para el consumo de la población, de la generación de oportunidades de trabajo y siendo amigable con la naturaleza.

El propósito del presente modelo de negocio es proporcionar orientaciones técnicas para el emprendimiento en mujeres campesinas del sector rural del cantón Guaranda, en el marco de la implementación de una empresa dedicada a la producción y comercialización de quesos que satisfaga la necesidad de consumo de quesos, al mismo tiempo que el de brindar una oferta amplia y variedad de productos lácteos, considerando las cualidades nutritivas que estos tienen para la salud. Además, poseen un mayor desempeño de crecimiento, lo que es favorable en el mercado, tanto local, nacional e internacional. Por último, son productos que brindan posibilidades de ser personalizados, con lo que se facilita su lanzamiento y su posicionamiento en el mercado; todo lo cual los convierten en una excelente fuente de generación de empleo y ganancias.

\section{Materiales y Métodos}

En la práctica, los proyectos de cooperación para el desarrollo, sean estos independientes o impulsados por organizaciones no gubernamentales de desarrollo, deben construir una base de datos con información cuantitativa y cualitativa (Hernández-Sampieri, Fernández y Baptista, 2014; Britton et al., 2017) relevantes acerca de los aspectos que se plantean en los objetivos del estudio, en la formulación de las estrategias, en los resultados formulados y ejecutados, a partir de indicadores que faciliten su formulación y evaluación (Vázquez, Torres-Jiménez and Caldentey, 2015).

La investigación que requirió la presente propuesta contó con una muestra de 389 habitantes de un universo total de habitantes de 216000 (INEC, 2010a). La población que conformó la muestra fue seleccionada a partir de un muestreo aleatorio, con un nivel de confianza del 95\%, según Scheaffer, Mendenhall y Ott (2019).

Para la obtención de la información primaria se utilizó la encuesta como instrumento de apoyo, la cual estuvo conformada de 32 preguntas adecuadas al contexto sociodemográfico y económico de la muestra objetivo. Para la agrupación de datos se estableció un criterio de similitud de las respuestas, que se codificaron para conseguir agrupaciones homogéneas en la información, utilizando el programa Python. Previamente, se hizo el estudio de correlación de las variables y un análisis de normalización de los datos, para, posteriormente, realizar las agrupaciones por variables y de mapas de calor para los individuos.

\section{Resultados y Discusión}

La creación de empresas pretende apoyar el desarrollo de la cadena de valor de los productos agrícolas. Asimismo, la materia prima que requiere la leche para su producción establece una secuencia de pasos que van desde su concepción, pasan por las fases intermedias de producción y llegan hasta la comercialización entre sus consumidores finales y su disposición final (Nutz and Sievers, 2016). Dentro de la empresa, la innovación se refiere a la implementación de tecnología, técnicas o herramientas que generan un cambio positivo y mejoran los procesos productivos; dicho de otra forma, es lo que facilita la eficiencia, la eficacia, la productividad y el desempeño viables y socialmente aceptables (López et al., 2019).

La relación entre ciencia, resolución de problemas de la vida diaria y bienestar de la mujer tienen reducida atención por parte de los científicos. Sin embargo, métodos como los de la observación cualitativa y las entrevistas semiestructuradas multinivel son herramientas que 
permiten interpretar las mejoras que se han presentado en las condiciones de calidad de vida, en la voluntad y en la motivación de quienes participan en la muestra (Pérez and Lutsak, 2017). Todo lo cual permite aportar con evidencias complementarias la construcción de la línea base de los factores relevantes en el desarrollo vivencial dentro de la zona demarcada por el proyecto, lo que favorece su comprensión.

Según datos de la FAO (2019), los productores de alimentos a pequeña escala producen casi el $80 \%$ de la leche en los países pobres. Por otro lado, de acuerdo con Oddone y Padilla Pérez (2017), la producción nacional de leche es de 1.600.000 litros diarios. El 54,1\% se utiliza en la fabricación artesanal de quesos; el $16 \%$ se destina al consumo industrial de grandes procesadoras; el 19,9\% se utiliza para el autoconsumo (consumo en fincas), el 5,5\%, para el consumo fresco (bidón) y un $4,4 \%$ se lo usa como materia prima de las fábricas de yogur y dulces. Además, dentro del estudio en la cadena de valor de la leche, los intermediarios no favorecen la producción de productos elaborados (Mercado, 2021). Esto no solo desincentiva los espacios cooperativos y la conformación de centros de acopio, sino que afecta de manera indirecta la calidad de la leche, ya que los intermediarios la venden a los procesadores de quesos, que, según su escala, tienen menores exigencias que la industria procesadora (Ministerio de Agricultura, Ganadería, 2016; Zambrano et al., 2018).

De acuerdo a la información del Banco Central del Ecuador (BCE, 2019), el destino principal de la producción de leche en el 2018 fue la comercialización (73\%); para la elaboración de quesos y quesillos se destinó el 25\%; y, para consumo familiar, el 2\%. El principal canal de mercadeo correspondió a los intermediarios, cuya participación alcanzó el $72 \%$; seguido por la venta directa a las plantas procesadoras, que representó el $15 \%$ de la comercialización; $\mathrm{y}$, finalmente, el 13\%, que se dio a través de la venta directa en los mercados locales. En todo caso, la producción de leche mantiene el rendimiento en 11 litros por vaca diarios, volumen similar al registrado en el mismo período del año 2017. Según el Ministerio de Agricultura y Ganadería, la Agencia de Regulación y Control Fito y Zoosanitario, Sistema de Fiebre Aftosa (SIFAE) en el 2020, en la provincia de Bolívar, de un total de 41711vacas en ordeño, se obtuvieron 202292 litros de leche, los que se distribuyen en ventas por litro, consumo en UPA (procesada dentro de la UPA) y alimentación al balde, para la alimentación de los terneros (Ministerio de Agricultura y Ganadería, 2019a).

Debemos aclarar que, según FAO-ODS_CELAC (2020), "las cualidades nutricionales del queso, es (sic) de gran importancia para el consumo humano, por lo que se considera un producto básico en la canasta básica familiar ecuatoriana". Esto genera una dinámica que se promueve, principalmente, su producción y comercialización (Ministerio de Agricultura, Ganadería, 2016), y, en especial, de quesos y otros derivados lácteos, facilitando con ello la generación de valor agregado favorable para el consumidor final. Esta dinámica propicia un crecimiento microempresarial que se presenta como una alternativa esencial para brindar precios justos, con calidez y calidad. Este encadenamiento constituye una ventaja para un número creciente de consumidores y como una estrategia de diferenciación para el productor (FMI, 2021; da Silva et al., 2013).

Los resultados mostraron una mayor presencia de mujeres $(51.2 \%)$; por otro lado, el $27,9 \%$ se encuentra en un rango de edad comprendido entre 25 a 30 años. E1 50.7\% son solteros y el $31.1 \%$ se encuentra cursando estudios universitarios. El 80,9\% vive en el sector urbano. Todos los encuestados conocen más de cuatro marcas de queso, incluido el queso fresco criollo.

La observación representativa de las variables de estudio nos permite establecer similaridades o disimilaridades entre las variables e individuos, lo que permite evidenciar la 
variabilidad conjunta y tipificar lo que sucede dentro de los datos. Dentro de las fuentes de información, para determinar la relación entre las variables, la estimación a partir de la Correlación de Pearson, nos muestra, acorde a la Figura 1, que los valores positivos más relevantes de las correlaciones corresponden a la Calidad-Forma del producto y Tamaño-Precio (0.8), además de las correlaciones negativas significativas Sexo-Actividad, Nivel de educación-Actividad, Consumo-Precio del Queso, Frecuencia de consumo-Precio (-0.15), lo que demuestra que existiría una relación inversa entre las variables.

\section{Figura 1}

\section{Correlación de las variables del estudio}

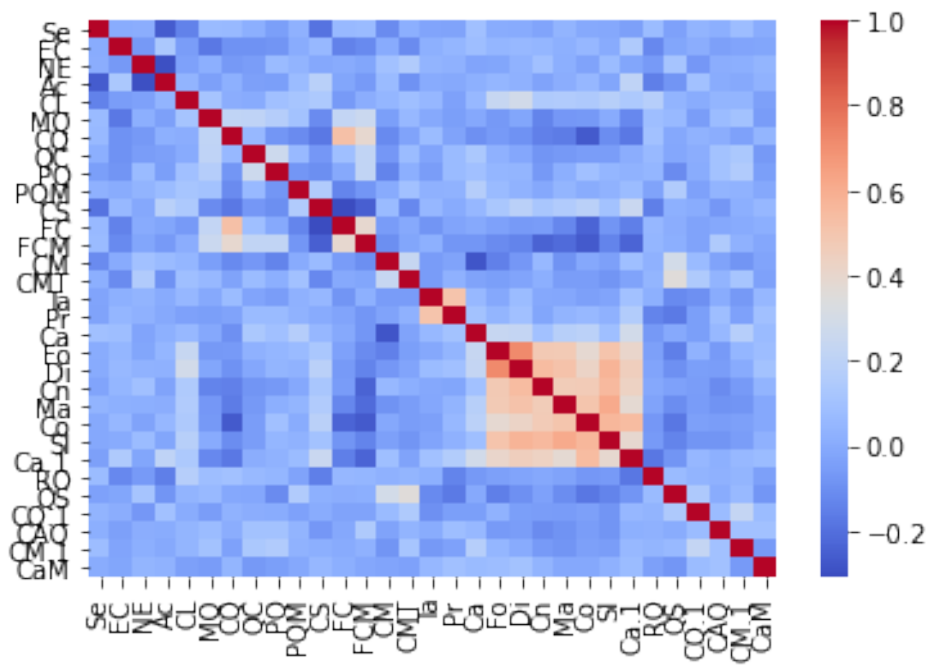

Fuente: Investigación 2021

Para la evaluación de los 389 datos, divididos en 2 conglomerados, se consideraron las variables con mayor aporte para el modelo, como se muestra en la Figura 2. La primera correspondió a las características sociodemográficas (Sexo, Edad, Estado civil, Nivel de Educación y Actividad Económica) y la segunda, a las del mercado (Consumo de Lácteos, Marca de queso, Consumo de queso, Marca de queso y precio de queso). Los dos últimos del primer grupo, a una distancia de 24 , podrían formar un subconjunto, mientras que, a una distancia de 18, los tres últimos del segundo grupo podrían conformar otro subgrupo.

\section{Figura 2}

\section{Cluster Jerárquico Truncado Empresarial}

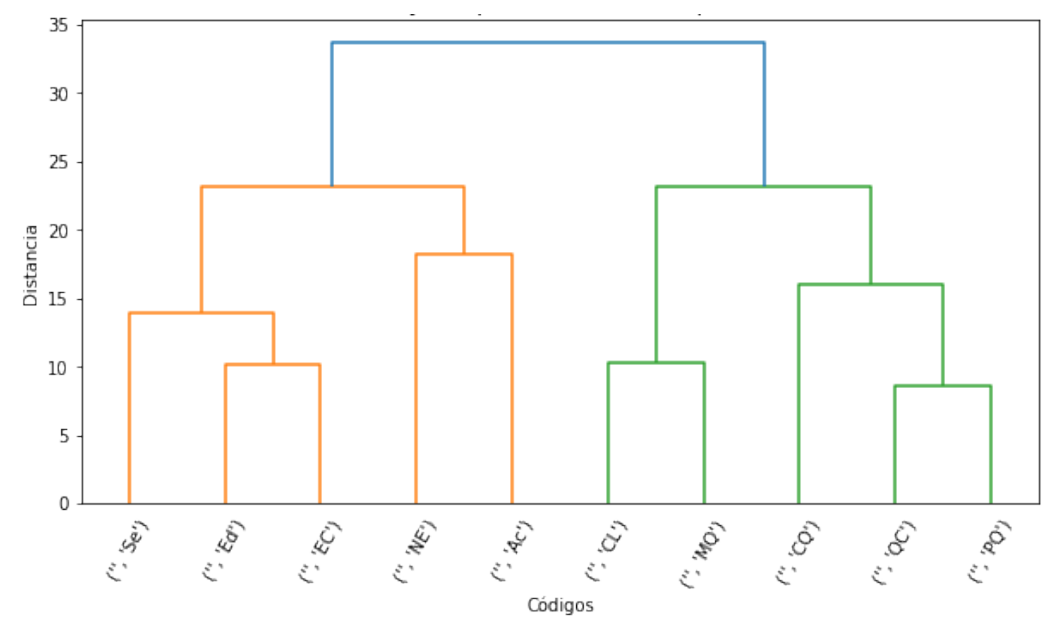

Fuente: Investigación 2021 
Tal como lo indica la Figura 2, a partir del dendrograma, que utilizó el método de Enlace "Ward", según el total de las observaciones, se obtuvieron dos agrupaciones de similitud. Dentro del primer grupo, con una división de 50, se distinguen dos grupos con una similitud de 25 y 30, respectivamente. Para, posteriormente, subdividirse en el primer caso a $\operatorname{los} 24,22,18,14$, según los niveles de corte respectivos. En sentido opuesto, en el grupo 2 se tienen subgrupos significativos, que se dividen a los 32, 27, 21, 19, y, posteriormente, subgrupos semejantes, con rendimientos mínimos para todas las observaciones.

Según el mapa de calor (Figura 3), siguiendo el degradado de calor, los colores más claros tienen un valor de 1 , en tanto el color azul oscuro, el valor de cero. Los valores intermedios toman diferentes tonalidades, requeridos para la visualización de los datos multidimensionales. En este gráfico es más notorio las similitud o distancia de las agrupaciones jerárquicas en el cluster, según las variables y las observaciones. Cada perfil indica las características de los individuos.

\section{Figura 3}

\section{Mapa de calor de las variables}

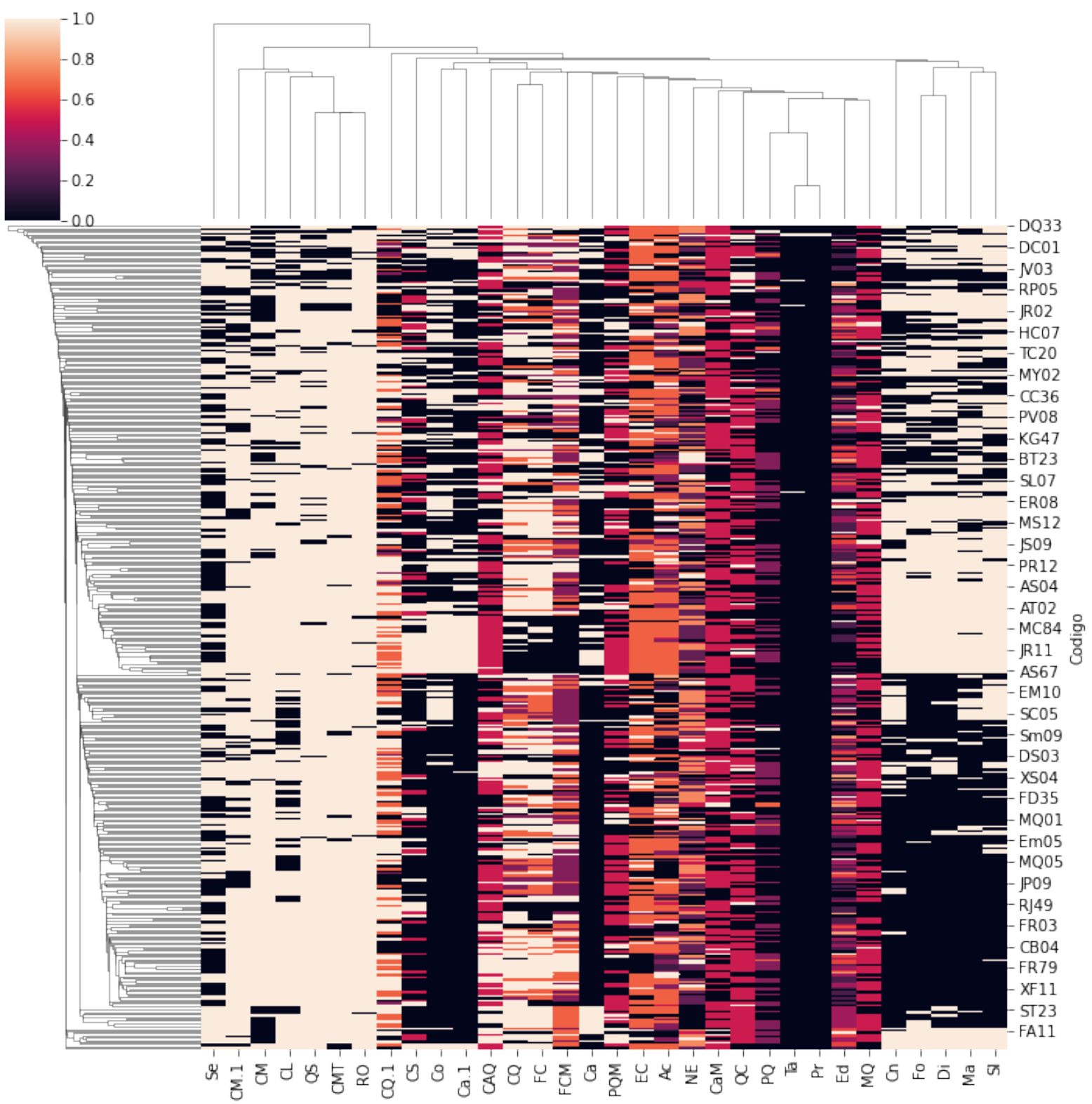

Fuente: Investigación 2021 
Para la conformación de la inversión y el financiamiento de la propuesta del proyecto, se recurrió a la búsqueda de información primaria y secundaria de fácil acceso y a la disponibilidad de equipos y materiales en el mercado, ofertados por los principales proveedores y distribuidores de productos necesarios para la conformación de la empresa. A continuación, se mencionan de manera resumida las inversiones y los presupuestos principales proyectados para la ejecución del proyecto en un tiempo de seis años (Tabla 1 y Tabla 2).

\section{Tabla 1}

Inversiones y financiamiento del proyecto

\begin{tabular}{|c|c|c|}
\hline \multicolumn{3}{|l|}{ INVERSIONES Y FINANCIAMIENTO } \\
\hline \multicolumn{3}{|l|}{ INVERSIONES } \\
\hline ACTIVOS FIJOS & VALORES & PORCENTAJE \\
\hline Terrenos & $\$ 8,000.00$ & $5.80 \%$ \\
\hline Edificios & $\$ 42,509.40$ & $30.79 \%$ \\
\hline Maquinaria y Equipos & $\$ 61,920.00$ & $44.85 \%$ \\
\hline Equipos de Computación & $\$ 1,650.00$ & $1.20 \%$ \\
\hline Muebles y Enseres & $\$ 2,970.00$ & $2.15 \%$ \\
\hline TOTAL ACTIVOS FIJOS & $\$ 117,049.40$ & $84.79 \%$ \\
\hline \multicolumn{3}{|l|}{ ACTIVOS DIFERIDOS } \\
\hline Gastos de Instalación & $\$ 2,500.00$ & $1.81 \%$ \\
\hline Capacitación & $\$ 500.00$ & $0.36 \%$ \\
\hline Gastos Legales de Constitución & $\$ 2,000.00$ & $1.45 \%$ \\
\hline Viajes (Contactos con Proveedores/clientes) & $\$ 1,000.00$ & $0.72 \%$ \\
\hline TOTAL ACTIVOS DIFERIDOS & $\$ 6,000.00$ & $4.35 \%$ \\
\hline \multicolumn{3}{|l|}{ CAPITAL DE TRABAJO } \\
\hline Capital de operación & $\$ 15,000.00$ & $10.87 \%$ \\
\hline TOTAL, INVERSIÓN & $\$ 138,049.40$ & $100.00 \%$ \\
\hline \multicolumn{3}{|l|}{ FINANCIAMIENTO } \\
\hline Crédito & $\$ 100,000.00$ & $72.44 \%$ \\
\hline Capital Social & $\$ 38,049.40$ & $27.56 \%$ \\
\hline TOTAL FINANCIAMIENTO & $\$ 138,049.40$ & $100.00 \%$ \\
\hline
\end{tabular}

Fuente: Investigación 2021

\section{Tabla 2}

Resumen de la Evaluación Financiera

\begin{tabular}{|l|l|}
\hline Tasa Mínima Aceptable de retorno & $16 . \%$ \\
\hline Valor Actual Neto & 206374.82 \\
\hline Tasa Interna de retorno & $15.56 \%$ \\
\hline Relación Beneficio-Costo & $\$ 2.49$ \\
\hline Punto de equilibrio en unidades & 70959 \\
\hline Punto de equilibrio en valores & $\$ 248356.40$ \\
\hline Recuperación de la inversión & 4.154 \\
\hline
\end{tabular}

Fuente: Investigación 2021

De manera ejecutiva, se realizó un modelo de prefactibilidad para conocer la viabilidad del proyecto de creación de una quesera, cuyo objetivo es diseñar un modelo de negocios para microempresarias dispuestas a invertir en este segmento de la cadena de valor de la leche. Para el modelo, se levantó mediante encuestas información orientada a identificar las necesidades 
de consumo de los clientes y colaboradores, como primer paso para determinar la oferta y demanda del producto. Además, se realizaron cuestionarios para el levantamiento de información in situ, así como se hizo una revisión bibliográfica para conocer variables socioeconómicas de la población cercana al lugar de donde se va a implementar la empresa. En base a los resultados obtenidos, se determinó la existencia de una demanda insatisfecha de queso en el mercado. La propuesta tiene por objetivo producir diferentes clases de queso, como el andino, y de sabores exóticos. La demanda de leche para la producción de 200 kilos de queso /día, con un promedio de 7 litros de leche por queso, se estima en 1500 litros de leche/día. Se concluye que el modelo de negocios propuesto de acuerdo a una evaluación financiera cuya rentabilidad se basa en una tasa de interés del 16\% anual, puede llegar a obtener una Tasa Interna de Retorno (TIR) de 15.56\%, Valor Actual Neto (VAN) de \$205874.92, CostoBeneficio (B-C) de $\$ 2.49$, es decir, por cada dólar invertido en la empresa se genera una ganancia de $\$ 1.49$ unidades monetarias. El periodo de recuperación de la inversión sería de 4 años y dos meses. Por otra parte, se recomienda a los futuros inversionistas la compra de acciones de la empresa, puesto que esto permitiría evaluar los ingresos y egresos proyectados de manera rentable.

Para el análisis FODA de la empresa, se consideraron factores que podrían afectar el desempeño de la empresa, así como aquellos podrían mejorarlo. A continuación, se detallan estos aspectos (Tabla 3).

Tabla 3

Análisis FODA de la microempresa

\begin{tabular}{|c|c|}
\hline Fortalezas & Oportunidades \\
\hline $\begin{array}{l}\text { - Desarrollar una microempresa asociativa, preferentemente de } \\
\text { mujeres } \\
\text { - Nuestros productos contienen un alto valor nutritivo } \\
\text { - Valores empresariales, liderazgo, apoyo organizativo } \\
\text { - Busca la fidelización de los clientes y proveedores de materia } \\
\text { - prima y productos } \\
\text { - Marca nueva en el mercado alimenticio } \\
\text { - Producto y presentación de marca de alta calidad } \\
\text { - Nueva variedad de productos con sabores exóticos } \\
\text { - Contacto personalizado con el cliente } \\
\text { - Cumplimiento de las normas de Bioseguridad } \\
\end{array}$ & $\begin{array}{l}\text { - Alianzas con microempresarios de leche de la zona } \\
\text { - Competencias y habilidades del equipo de trabajo } \\
\text { - Crecimiento anual } \\
\text { - Redes sociales como marketing } \\
\text { - Ubicación del local en el centro de la ciudad } \\
\text { - Producto con empaque ecológico } \\
\text { - Interacción con el cliente } \\
\text { - Creciente tendencia al consumo de alimentos nutritivos } \\
\text { - Apoyo a la cadena de valor de la leche } \\
\text { - Reactivación de la economía local }\end{array}$ \\
\hline Debilidades & Amenazas \\
\hline $\begin{array}{l}\text { - Riesgo en la comercialización, hasta el posicionamiento } \\
\text { efectivo de la marca } \\
\text { - Riesgo de accidentes y mala producción inicial } \\
\text { - } \text { Alto riesgo financiero } \\
\text { Riesgo de producción, al ser un producto con nuevas } \\
\text { características } \\
\text { Adaptación al mercado, nuevo emprendimiento }\end{array}$ & $\begin{array}{l}\text { Incremento de productores informales de quesos en el sector } \\
\text { Empresas comunitarias } \\
\text { Sinches. }\end{array}$ \\
\hline
\end{tabular}

Fuente: Investigación 2021

En la actualidad a nivel nacional no se encuentran datos históricos del consumo de lácteos, sus derivados, en especial, quesos y sus preferencias, por lo que la proyección progresiva de la oferta y demanda del proyecto se la hace considerando a la población histórica del cantón, recogida en los datos del censo del 2010 (INEC) y del PDOT del cantón Guaranda y la provincia y datos referenciales de la encuesta relacionados con el consumo, la compra del producto y la disponibilidad en el mercado.

En el contexto de la pandemia del COVID 19, las oportunidades de las microempresas y de la reactivación de su economía, dependen de las entidades crediticias públicas o gestoras de crédito de financiamiento, que utilizan fondos provenientes del presupuesto general del Estado o de Organismos multilaterales. Estas fuentes de financiamiento suelen dirigir su inversión a la producción de materia prima local y a la sustitución de bienes importados por 
materia prima o productos elaborados dentro del país, según lo dispone la Ley Orgánica de Apoyo Humanitario (SENPLADES, 2020).

Hasta el 2020, la industria láctea ha presentado un alto crecimiento en Ecuador, según el estudio de tendencias de consumom. Esto debido en parte al establecmiento de la semaforización que permite la identificaicón de productos nutritivos, además, de otros factores, como algunos cambios que se han registrado en la matriz productiva, y que fomentan el desarrollo de este tipo de industrias. En los últimos años, se ha registrado un incremento anual de $25 \%$ y hasta $30 \%$ en el consumo de leche y de sus productos derivados según reportes de la Universidad de Cuenca y de las empresas dedicadas a la producción láctea (Mipro, 2020; AEI_PNUD, 2018; FAO, 2019).

Las iniciativas económicas en las actuales circunstancias tienen un efecto amortiguador, es decir, ayudan a mejorar el equilibrio durante el estado de alarma mundial frente al Covid-19. Esto se constata en una creciente demanda de los productos, especialmente, frescos, lo que ha permitido un amplio desarrollo de la economía formal. Por otro lado, en la economía informal la escases de iniciativas han modificado la distribución o, en algunos casos, la capacidad de asociarse entre productos agrícolas, originando una gran incertidumbre a la hora de invertir en actividades vinculadas al abastecimiento (Escribano y Hummel, 2020). En definitiva, contextos como estos son una oportunidad para la constitución de empresas que permitan fomentar la reactivación de los pequeños productores, quienes abastecen en su mayor parte de productos al mercado local y nacional.

También el estudio, ha permitido evidenciar que, en la provincia de Bolívar, el 38,8\% de la Población Económicamente Activa (PEA), de un total de 72.158 habitantes (INEC, 2010b), realiza actividades vinculadas a la agricultura y a los trabajos calificados, lo que permite contar con recursos humanos, naturales y productivos suficientes para el fortalecimiento de las cadenas productivas y los productos no tradicionales, que realizan el mayor aporte a la economía nacional, luego de la producción de derivados de petróleo.

Tomando el ejemplo de Uruguay, por Decreto se introdujo en la matriz de evaluación de proyectos de inversión modificaciones normativas para promover proyectos innovadores que incorporen el componente ambiental, así como cambios en el régimen de promociones ante diferentes escenarios económicos (Capurro et al., 2020). Por otro lado, en Ecuador se realizan modificaciones a las leyes con respecto a la reactivación económica y a la evaluación de proyectos por parte de las entidades financieras. Esto con el objetivo de promover seguros agrícolas que mejoren la sostenibilidad y sustentabilidad de las empresas a futuro.

En el contexto social de la cultura maya, por ejemplo, las mujeres indígenas se han organizado en torno al trabajo colectivo de la producción agroecológica. En la actualidad, su lucha en todos los campos, políticos y productivos, han mejorado sus condiciones sociales, alternando el poder y el conocimiento (Leyva, 2018). Las mujeres en Ecuador se vienen proyectando de forma progresiva con la conformación de asociaciones que fortalecen sus capacidades en todos los niveles, así como promueven la conservación de la paccha mama y una colaboración permanente dentro de sus organizaciones. Es evidente que el fortalecimiento de las capacidades de la mujer debe ser revisado por completo en la política pública para el fortalecimiento de los planes de desarrollo nacional, pues la mujer es un componente principal en el desarrollo productivo del país.

Un aspecto que se debe considerar a la hora de iniciar un negocio es la Responsabilidad Social Empresarial (RSE). En este sentido. el Observatorio Empresarial de la Universidad del 
Azuay considera que las mujeres están más dispuestas al cambio en el ámbito empresarial. En opinión de los consumidores, son las mujeres las que deben comunicar la gestión administrativa, ambiental y social de la empresa en la localidad (Azuay, 2019). Estos aspectos han sido considerados para evaluar la matriz FODA empresarial de esta propuesta, ya que la mujer es quien administra los recursos en el campo. Este aspecto se debe considerar también en la difusión de la marca y en quienes se encuentran bregando por el reconocimiento de los empresarios y emprendedores, pues se enfrentan cada día a las condiciones de un mercado exigente que demanda responsabilidades sociales de la empresa.

Posterior a la crisis mundial del COVID 19, los mayores impactos se observarán con el aumento de la desigualdad y el incremento de los problemas relacionados con la salud y la situación de los adultos mayores, los jóvenes desempleados, así como de las mujeres migrantes (CEPAL, 2020). Una de las funciones que el campo cumple es la de abastecer de productos a la ciudad. En este contexto, el queso constituye un componente principal de la canasta básica de los hogares ecuatorianos, por lo que su producción y comercialización goza de esta ventaja. Cabe enfatizar que, si se cuenta con un local comercial para la venta directa del producto, así como si se entrega de forma directa el producto, se podría evitar el uso de intermediarios. Además de estos elementos, también se deberían imaginar diversas formas novedosas para atraer a los consumidores: hacer entregas con el denominado delivery, o promociones utilizando las redes actuales, como el tiktokers.

El factor negativo al que se podría estar enfrentando este negocio, es la creciente migración de la mujer, lo que se puede evidenciar, sobre todo, en algunos sectores del país. Asimismo, el ingreso de migrantes de otros países que buscan oportunidades en nuestro entorno, bajo condiciones de remuneración más bajo que el que recibe un trabajador local. Un ejemplo de lo que se describe aquí, corresponde a la migración de mujeres marroquíes que, últimamente, han llegado a ciertas zonas rurales de España para trabajar en la primera fase agrícola de la producción de frutos rojos (Garcés and Güel, 2021). De manera similar, la heterogeneidad de los mercados formales, compuesto de mujeres, indígenas, afrodescendiente micro y pequeños empresarios en situación de empleo precario, demandan de una respuesta inmediata de los centros políticos, quienes deben garantizar el acceso a los servicios, al crédito y a la capacidad de generar ingresos (Alzúa and Gosis, 2020).

\section{Conclusiones}

La presente investigación ha mostrado la incidencia que ha tenido el estado de emergencia provocado por el COVID 19 en las iniciativas agroproductivas del sector periurbano de Guaranda. En este contexto, se ha demostrado el papel fundamental que tiene la producción agrícola en la economía local y provincial, y el potencial que tienen los diferentes escenarios dentro de los contextos socioeconómicos locales. El estudio de caso del modelo agroproductivo, junto con las economías del mercado, son favorables como una forma de reactivación económica para el sector formal, como una forma de abastecimiento de los productos de la canasta básica nacional.

Dentro del economía social y solidaria, en la perspectiva de muchos proyectos para fomentar la economía social, no se han incluido consideraciones relacionadas con el entorno territorial, lo que ha significado excluir de sus enfoques la lógica de las acciones colectivas, la preservación de los principios y valores sociales frente a los riesgos económicos externos. La adopción de modelos de gestión provenientes de modelos externos es el resultado de la idealización de programas o proyectos no operativos dentro del sector en los que estos se quieren implementar. 
En el contexto de la inserción económica del Estado en el escenario sociopolítico, se ha observado que un impedimento para lograr la formalización de las iniciativas agroproductivas vinculadas a la mujer campesina es el endurecimiento de los requisitos legales en la constitución de empresas.

Estas conclusiones se suman al debate de la importancia del rol que tiene la mujer en los emprendimientos y en la innovación dentro de la economía local formal. La perspectiva sociológica y antropológica resultan importantes para entender la complejidad de las relaciones que se establecen entre la mujer, el estado y el crecimiento de la economía campesina. Por este motivo, desde la política pública, se requiere impulsar la articulación de las dimensiones sociales, políticas, económicas, ecológicas, productivas en el fortalecimiento de los proyectos innovadores de emprendimiento, y comprometer a organismos públicos para implementar y capacitar prácticas amigables con la naturaleza, especialmente, en los procesos de conservación de los recursos naturales.

\section{Referencias}

AEI_PNUD. (2018). El ABC del emprendedor: guía práctica para arrancar con tu negocio (A. para el E. y la Innovación (ed.)). https://www.ec.undp.org/content/ecuador/es/home/library/reduccion-de-lapobreza/el-abc-del-emprendedor.html

Alzúa, L., \& Gosis, M. (2020). Impacto Social y Económico de la COVID-19 y Opciones de Políticas en Argentina. PNUD América Latina y El Caribe, 6, 1-27. www.latinamerica.undp.org

Arteaga-Cruz, E. L. (2017). Buen Vivir (Sumak Kawsay): definiciones, crítica e implicaciones en la planificación del desarrollo en Ecuador. Saúde Em Debate, 41(114), 907-919. https://doi.org/10.1590/0103-1104201711419

Azuay, U. del. (2019). Observatorio Empresarial- Segundo Boletín. Casa Editora, 1(1), 1-134. https://doi.org/ISSN: 2661-6823

Banco Central del Ecuador. (2019). Reporte de Coyuntura Sector Agropecuario: Vol. IV (www. bce.ec, Issue 91).

http://www.economia.gob.mx/files/comunidad_negocios/industria_comercio/informacionSectorial/min ero/reporte_coyuntura_mineria_nacional_0514.pdf

Banco Interamericano de Desarrollo. (2004). El Marco Lógico para el Diseño de Proyectos.

Barrientos, P. (2019). Estrategia de integración del pequeño agricultor a la cadena de exportaciones. Semestre Económico, 22(51), 83-123. https://doi.org/10.22395/seec.v22n51a5

Britton, A., Chu, J. I., Vergara de Caballero, E., Ríos Castillo, I., \& Barría, M. (2017). Estudio piloto de preferencia y aceptabilidad de recetas con camote biofortificado: un estudio transversal a nivel comunitario. Perspectivas En Nutrición Humana, 19(2), 137-150. https://doi.org/10.17533/udea.penh.v19n2a02

Capurro, A., Deagosto, G., Ithurralde, S., \& Oddone, G. (2020). Impacto Social y Económico de la COVID-19 y Opciones de Políticas en Uruguay. Mayo, 10(10), 41. file://C:/Users/Usuario/Downloads/undp-rblacCD19-PDS-Number10-ES-Uruguay.pdf

CEPAL. (2020). América Latina y el Caribe ante la pandemia del COVID-19. Efectos económicos y sociales. Informe especial COVID-19, No1. In C. or. Www (Ed.), Informe Especial COVID-19 (Issue 1). https://www.cepal.org/es/publicaciones/45337-america-latina-caribe-la-pandemia-covid-19-efectoseconomicos-sociales

da Silva, C. A., Baker, D., Shepherd, A. W., Jenane, C., \& Miranda, S. (2013). Agroindustrias para el desarrollo. FAO. 
Deere, D. (2018). El derecho de la mujer a la tierra, los moviminetos sociales rurales y el estado en las reformas agrarias latinoamericanas del siglo XXI. In CLACSO-JSTOR (Ed.), La cuestión agraria y los gobiernos de izquierda en América Latina (p. 218). https://www.jstor.org/stable/j.ctvn96g0z.5\%0AJSTOR

Dos Santos, T. (2020). La revolución científica-técnica y la acumulación de capital. In CLACSO- JSTOR (Vol. 2, Issue 2). https://www.jstor.org/stable/j.ctv1gm024c.6

Escribano, P., \& Hummel, A. (2020). El papel de la economía informal en los proyectos agroecológicos durante el COVID-19 The role of the informal economy in agro-ecological projects during COVID-19. Espaco Aberto, 58, 437-461. https://doi.org/http://dx.doi.org/10.1590/S0104-71832020000300014 El

FAO-ODS_CELAC. (2020). Seguridad Alimentaria bajo la Pandemia de COVID-19. FAO, 25.

Figueroa, D., \& García, L. E. (2019). Comprensión de las acciones comunitarias en educación ambiental en Chiquinquirá, Boyacá. Praxis \& Saber, 10(23), 293-314. https://doi.org/10.19053/22160159.v10.n23.2019.9735

Fondo Monetario internacional. (2021). Actualizaciones de las perspectivas de la economía mundial. Fondo Monetario Internacional, 1-23.

Food and Agriculture Organization - FAO. (2019). El trabajo de la FAO en la agricultura familiar. Prepararse para el Decenio Internacional de Agricultura Familiar (2019-2028) para alcanzar los ODS. FAO. http://www.fao.org/3/ca1465es/CA1465ES.pdf

Garcés, B., \& Güel, B. (2021). Temporeros agrícolas en contexto de pandemia: nuevas caras de un viejo problema. Anuario CIDOB de La Migración, 1(5), 86-100. https://doi.org/DOI: doi.org/10.24241/AnuarioCIDOBInmi.2020.86

Generalitat Valenciana, D. G. de C. y S. (2017). Guía para elaboración de los estudios de Línea de Base de los Proyectos Subvencionados por la Generalitat 2017. In Guía Para Elaboración De Los Estudios De Línea De Base De Los Proyectos Subvencionados Por La Generalitat 2017.

http:/www.cooperaciovalenciana.gva.es/documents/164015995/164468323/Guía+para+la+elaboración + de+los+estudios + de+Línea+de+Base+de+proyectos+subvencionados+por+la+Generalitat+2017/df11 e7bb-049c-487f-8b09-57094c7bbf8b;jsessionid=FF1EEBBDB6E69C60DBEC78CB4455

Henríquez, G. R., Garzon, M. A., Mejia, C., Torrenegra, A. J., \& Rada, J. A. (2019). Medición De Impactos Socioambientales Y Responsabilidad Social Organizacional. Dimensión Empresarial, 17(4). https://doi.org/10.15665/dem.v17i4.2111

Hernández-Sampieri, R., Fernández, C., \& Baptista, P. (2014). Metodología de la Investigación (M. G. Hill (ed.); Sexta Edic).

HubSpot. (2019). Manual para crear la misión y visión de tu empresa. Hubspot, 4-37. https://cdn2.hubspot.net/hubfs/53/00-OFFERS-HIDDEN/\%5BSPANISH\%5D Mission and Vision/Ebook_MisiónYVisión_180419 v6.pdf?hubs_postcta=author\&hubs_post=blog.hubspot.es\%2Fmarketing\%2Fmision-vision-valoresejemplos\&hubs_offer=offers.hubspot.es\%2Fmision-y-vis

INEC-ESPAC. (2020). Encuesta de Superficie y Producción Agropecuaria Continua (ESPAC). ESPAC, 1-43. https://www.ecuadorencifras.gob.ec/documentos/web-inec/Estadisticas_agropecuarias/espac/espac2019/Presentacion de los principales resultados ESPAC 2019.pdf

INEC. (2010a). Resultados del Censo 2010 de población y vivienda del Ecuador. Fascículo Provincial Manabí. In Instituto Nacional de Estadística y Censos (pp. 1-7). http://www.ecuadorencifras.gob.ec/wpcontent/descargas/Manu-lateral/Resultadosprovinciales/manabi.pdf\%0Ahttp://www.inec.gob.ec/cpv/descargables/fasciculos_provinciales/manabi. pdf

INEC. (2010b). Resultados del Censo INEC 2010 de población y vivienda en el Ecuador. Fasciculo Provincia Bolívar. Inec, 8. http://www.ecuadorencifras.gob.ec/wp-content/descargas/Manu-lateral/Resultadosprovinciales/bolivar.pdf 
Kotler, P., \& Armstrong, G. (2017). Fundamentos de Marketing (Pearson (ed.); Decimoprim). https://www.academia.edu/8889213/Fundamentos_del_Marketing_Kotler_11va_ed?auto=download

Leyva, X. (2018). ¿Reconfiguración del régimen moderno de saber / poder en América Latina: dónde, cómo y quiénes? In Prácticas otras de conocimientos, p. 70. https://www.jstor.org/stable/j.ctvn5tzv7.6

López, J., Damián, M., Álvarez, J., Mendéz, J., Rappo, S., \& Paredes, J. (2019). Innovaciones radicales y progresivas en el manejo del maíz en Calpan, Puebla, México. Revista Mexicana de Ciencias Agrícolas, 10(2), 277-288.

MAGAP. (2017). Uso del Suelo (ha) por Categoría - 2017 Número de UPA por tamaño - III Censo Nacional Agropecuario 2000 Principales Cultivos - 2017 Ganado por Especie Nivel Provincial: LOS RÍOS Destino de la Producción Lechera - 2017 Nivel Provincial: Los Ríos Destin. 2017.

Martínez, D. (2015). Entre economía social y economía popular. Confusiones y desaciertos políticos en el "Ecuador del Buen Vivir". Eutopía - Revista de Desarrollo Económico Territorial, 7, 147. https://doi.org/10.17141/eutopia.7.2015.1669

Mercado, S. de C. de P. de. (2021). Estudio de Mercado NSCPM-IGT-INAC-002-2019 "Sector lácteo" Versión pública (S. de control de P. de Mercado (ed.)). https://www.scpm.gob.ec/sitio/wpcontent/uploads/2021/04/estudio_de_mercado_sector_lacteo_SCPM-IGT-INAC-002-2019.pdf

Ministerio de Agricultura, Ganadería, A. y P. (MAGAP). (2016). La politica Agropecuaria Ecuatoriana. Hacia el desarrollo territorial rural sostenible 2015-2025. I Parte (G. A. y P. Ministerio de Agricultura (ed.)).

Ministerio de Agricultura y Ganadería. (2019a). Bovinos_Agrocalidad.

Ministerio de Agricultura y Ganadería. (2019b). Panorama Agroestadístico. Octubre 2019. Panorama Estadístico Octubre 2019, 2018.

Ministerio de Turismo del Ecuador. (2020). Diseño Del Plan Estratégico De Desarrollo De Turismo Sostenible Para Ecuador "Plandetur 2020" Informe Final. Tourism\&Leisure, 536. http://www.turismo.gob.ec/wpcontent/uploads/downloads/2013/02/PLANDETUR-2020.pdf

Mipro. (2020). Visión agroindustrial. http://servicios.produccion.gob.ec/siipro/downloads/temporales/8_Vision Agroindustrial 2025.compressed.pdf

Moreno, C., Molina, J. I., Ortiz, J., Peñafiel, C., \& Moreno, R. (2020). The value chain of tree tomato (Solanum betaceum) network in Ecuador. Agronomy Mesoamerican, 31(1), 13-29. https://doi.org/10.15517/AM.V31I1.36887

Nutz, N., \& Sievers, M. (2016). Guía General para el Desarrollo de Cadenas de Valor. https://www.ilo.org/wcmsp5/groups/public/---ed_emp/---emp_ent/--ifp_seed/documents/instructionalmaterial/wcms_541432.pdf

OCDE/FAO. (2019). OCDE-FAO Perspectivas Agrícolas 2019-2028 Enfoque especial América Latina (O. Publishing (ed.)). Organización de las Naciones Unidas para la Alimentación y la Agricultura (FAO).

Oddone, N., \& Padilla Pérez, R. (2017). Fortalecimiento de cadenas de valor rurales. Cepal, 444. https://repositorio.cepal.org/bitstream/handle/11362/42077/S1700166_es.pdf

Ortegón, E., Pacheco, J., \& Prieto, A. (2015). Metodología del marco lógico para la planificación, el seguimiento y la evaluación de proyectos y programas (CEPAL (ed.)). www.cepal.org/es/suscripciones. www.cepal.org/es/suscripciones

Pérez, M. and Lutsak, N. (2017) 'La producción científica sobre la innovación social para el desarrollo local. Una revisión bibliométrica', Revista Prisma Social, 0(19), pp. 146-182.

Pizaña, H., Fletes, H., \& González, A. (2019). Tema central la Frailesca: vulnerabilidad y resistencias *. Eutopía. Revista de Desarrollo Económico Territorial, 15. https://doi.org/http://doi.org/10.17141/eutopia.15.2019.3865 
Ramirez, E. (2007). Manual Para Elaboración Línea De Base En Proyectos FOS. In Rimisp (Vol. 1, p. 13). http://rimisp.org/wp-content/files_mf/135937839217.pdf

Scheaffer, R., Mendenhall, W., \& Ott, L. (2019). Elementos de Muestreo (G. E. Iberoamérica (ed.); Third Edit).

SENPLADES. (2020). Ley Orgánica de Apoyo Humanitario. Registro Oficial Semplades, 1-13. http://www.planificacion.gob.ec/zonas-distritos-y-circuitos/

Territorio Ecuador ODS. (2018). Los ODS en Ecuador: Rol del Estado en su implementación. In Boletin Informativo Panorama Sostenible (Tomo3.). Quito:ODS Territorio Ecuador.

Valencia Cruzaty, L. (2017). Emprendimiento en Ecuador. Estudio de Caso: Provincia de Santa Elena. Killkana Social, 1(2), 27. https://doi.org/10.26871/killkana_social.v1i2.36

Vanoni, G., Omaña, A., Perez, O., Brito, M., Arenas, A., \& Vasco, F. (2017). Casos de Marketing en el entorno empresarial ecuatoriano (Primera). Universidad de Los Andes.

Varisco, C. (Universidad N. de M. del P.-A. (2016). Turismo Rural: Propuesta Metodológica para un Enfoque Sistémico. Revista de Turismo y Patrimonio Cultural, 14, 153-167.

Vázquez, M. J., Torres-Jiménez, M., \& Caldentey, P. (2015). Límites del marco lógico y deficiencias de la cooperación al desarrollo para medir el impacto. Revista Iberoamericana de Estudios de Desarrollo, $4(2), 1-26$.

Vergara, L., \& Kay, C. (2018). Agronegocio, campesinos, estado y gobiernos de izquierda en América Latina: Introducción y reflexiones teóricas. In JSTOR (Ed.), La cuestión agraria y los gobiernos de izquierda en América Latina. CLACSO. https://www.jstor.org/stable/j.ctvn96g0z.4

Zambrano, J., Barrera, V., Murillo, I., \& Domínguez, J. (2018). Plan Estratégico de Investigación y Desarrollo Tecnológico del INIAP 2018-2022. Innovando el Agro Ecuatoriano. www.iniap.gob.ec 